\title{
RISCO E RESILIÊNCIA PSICOSSOCIAL: DESAFIOS NA PRÁTICA DA VELA
}

\author{
Priscilla Pinto Costa da Silva \\ Universidade Federal da Paraíba, João Pessoa, Paraíba, Brasil \\ Emília Amélia Pinto Costa da Silva \\ Universidade Federal do Paraná, Curitiba, Paraná, Brasil \\ Andrea Maria Pires Azevedo \\ Universidade de Brasília, Brasília, Distrito Federal, Brasil
}

Samara Queiroz do Nascimento Florêncio

Centro Universitário de João Pessoa, João Pessoa, Paraíba, Brasil

Leonardo dos Santos Oliveira

Universidade Integrada de Patos, Patos, Paraíba, Brasil

Clara Maria Silvestre Monteiro de Freitas

Universidade de Pernambuco, Santo Amaro, Pernambuco, Brasil

\section{Resumo}

O objetivo deste estudo foi analisar fatores de resiliência a partir de situações de risco na prática da vela. A pesquisa de campo envolveu 19 velejadores do Iate Clube da Paraíba, com idades entre 19 e 64 anos. Foram utilizados na coleta de dados, um questionário sociodemográfico e um roteiro de entrevista semiestruturada. As respostas foram transcritas e submetidas ao soft AQUAD 6 para a obtenção das categorias a partir da análise de conteúdo, sendo organizadas em diagramas. Os resultados apontaram para três categorias: sensações sem a prática da vela; sensações em situação de risco na prática da vela; e a resiliência na prática da vela. Observou-se que, nesta prática, houve avanços nos fatores emocionais, trazendo um ressiginificado para a vida. Assim, o equilíbrio emocional auxilia nas situações de risco encontradas no mar, conduzido pela melhoria dos fatores da resiliência.

Palavras chave: Risco. Resiliência. Vela.

\section{Introdução}

As situações de risco das práticas corporais realizadas na natureza despertam diferentes sensações, podendo ser divididas em dois polos: um 
positivo, referente às sensações prazerosas; e o outro negativo, que diz respeito às sensações como o medo, a ansiedade e o desespero. (MARINHO, 2008; 2009; LAVOURA; SCHWARTZ; MACHADO, 2007;LE BRETON, 2009; SILVA; FREITAS, 2010) Estas sensações podem despertar sentimentos de revelação pessoal (LE BRETON, 2006; 2007a; 2007b; 2009; BRUHNS, 2009), como também podem desenvolver traumas (COOKE et al., 2000).

A busca por novas sensações, a partir das práticas corporais realizadas na natureza, associa-se à simbologia da hiperrealidade (BOUDRILLARD, 2008). Desta forma, o sujeito é conduzido a entrar em um mundo fictício (MARINHO, 2009), onde o risco imaginário desperta a sensação de jogar com a morte (LE BRETON, 2009). O autor aponta que estas práticas conduzem à busca de novos significados na vida, os quais foram perdidos pelas transformações do cotidiano.

Os fatores de risco das práticas corporais realizadas na natureza são enfrentados como experiências adversas, mas que podem trazer resultados favoráveis, como a superação de obstáculos (SILVA; FREITAS, 2010). No cenário natural, como o mar, no caso da vela, as situações de risco enfrentadas requerem um estado psicológico equilibrado para que o sujeito tenha níveis significantes de tomada de decisão consciente e reverta o quadro sem prejudicar os resultados da tripulação (FERNANDES et al., 2007).

Estados emocionais, em velejadores, também são classificados em sensações positivas e negativas, como felicidade e tristeza (BRANDT et al, 2010), sendo que as negativas podem trazer maiores transtornos em situações de risco. Este raciocínio conduz o domínio da Teoria da Resiliência, apropriada pela Psicologia para compreender e superar situações antagônicas (WALLER, 2001). Neste sentido, o conceito de risco no desenvolvimento de programas de prevenção e intervenção, trata da capacidade de resposta adaptativa em face à adversidade, considerando indicadores de múltiplas variáveis (HAGGERTY, et al., 2000).

Seguindo os escritos de Fraser e Pakenham (2009), a Resiliência pode ser entendida como o processo em que os efeitos nocivos ou prejudiciais dos fatores de risco são mediados ou removidos por influência de fatores de proteção. Lacharite (2005) caracteriza a Resiliência como o aumento da probabilidade de um resultado positivo específico na presença de uma determinada situação de risco.

Nesta direção, Fraser e Pakenham (2009) apontam que os mecanismos de resiliência têm sido propostos como a melhoria dos resultados advindos de modificações da influência de fatores de risco, da interrupção de sequelas, da melhoria da autoestima e da promoção de oportunidades para o sujeito. Além disso, Dolbier, Jaggars e Steinhardt (2010) mostram em seus estudos a melhoria da resiliência quanto às variáveis: autoestima, autoliderança e crescimento pessoal - as quais foram avaliadas a partir de intervenções psicoeducacionais.

No que diz respeito ao esporte, a superação e as formas de lidar com a adversidade são frisadas constantemente. Desta forma, a Resiliência possibilita a capacidade de resistir ou recuperar-se rapidamente a partir de condições desfavorá- 
veis, mesmo diante de situações de risco ou adversidade (GALLI; VEALEY, 2007; 2008; SABISTON et al., 2010; Valle, 2007).

Estudos de Gould; Dieffenbach e Moffatt, (2002) realizados com campeões olímpicos, apontaram os principais fatores de resiliência que proporcionam equilíbrio na vida do atleta, destacando aspectos como a capacidade de lidar com a ansiedade e controle, confiança, resistência mental, capacidade de concentração e bloqueio de distração, competitividade, trabalho de ética, capacidade de estabelecer e alcançar metas, níveis elevados de esperança e otimismo. Nesta perspectiva, os equilíbrios psíquico e físico são fatores importantes e estão ligados ao apoio dos pais e treinadores para que se alcance o sucesso do atleta (LAVOURA; ZANETTI; MACHADO, 2008).

No âmbito da recreação e do lazer, pesquisas (ALLEN; HURTES; STEVENS, 1999; HURTES, et al., 2000; IWASAKI; MACTAVISH; MACKAY, 2005; PEACOCK-VILLADA; DECELLES; BANDA, 2007) mostram que as práticas realizadas auxiliam na eficácia de fatores de resiliência, como: habilidades individuais, nível de humor, independência, otimismo, relacionamento, criatividade, valores pessoais, e outros. Desta forma, as sensações negativas, como o estresse, podem ser aliviadas ou neutralizadas a partir da promoção do lazer (IWASAKI; MACTAVISH; MACKAY, 2005).

Nas práticas corporais realizadas na natureza, os estudos referentes aos riscos e resiliência, como exemplo os realizados pelos autores Moote e Wodarski (1997), apresentam um programa de prevenção para adolescentes em situações de risco, como vícios, ausência de familiares, estresse e problemas de comportamento. Outros estudos (BLOEMHOFF, 2006; GREEN; KLEIBER; TARRANT, 2000) avaliaram também adolescentes em situações de risco e trataram esta temática nas práticas com corda. Isso levou a melhorias no comportamento e de problemas emocionais, o que recai ao desenvolvimento da resiliência.

Contudo, são insuficientes os estudos realizados no âmbito da Educação Física e das práticas corporais realizadas na natureza, sobretudo em ambiente aquático, que envolvem situações de risco e resiliência. Sendo assim, o presente estudo tem o objetivo de analisar fatores da resiliência a partir de situações de risco na prática da vela.

\section{Metodologia}

Para viabilizar o desenvolvimento deste estudo, optou-se por realizar uma pesquisa descritiva, de campo, justificando sua importância no âmbito sociocultural, e desvendando constructos, por meio de ações e linguagens dos atores sociais envolvidos (BANKS, 2009; HOLLINSHEAD, 2006; LINCOLN; GUBA, 2003). Nesta direção, as Ciências Sociais e Culturais permitem descobrir e ampliar o conhecimento dos fenômenos de uma realidade social (HOLLINSHEAD, 2006). 
Os participantes selecionados, intencionalmente, foram 19 velejadores do Iate Clube da Paraíba, da cidade de João Pessoa - Paraíba - Brasil, com idades entre 19 e 64 anos, sendo que apenas um dos velejadores era do sexo feminino. O critério de inclusão foi o tempo de experiência e prática da vela, sendo selecionados aqueles com, no mínimo, cinco anos de prática. A opção por este critério diz respeito às experiências e às relações estabelecidas com os elementos da natureza, como o mar e o vento, bem como os conhecimentos técnicos, advindos das vivências.

Foram utilizados como instrumentos para a coleta de dados um questionário sociodemográfico e um roteiro de entrevista semiestruturada, por permitirem eficácia nas respostas e oportunizarem o aprofundamento de determinado tema (COHEN et al., 2000; DELGADO; GUTIÉRREZ, 1995). O questionário caracterizou o perfil dos participantes e a entrevista seguiu os critérios estabelecidos por Henderson (1991), iniciando pelas perguntas gerais, como o surgimento do interesse pela prática da vela, seguindo para as questões específicas, relacionadas com as transformações que a prática da vela trouxe para o cotidiano.

O trabalho de campo foi realizado aos sábados e domingos, conforme planejamento pré-estabelecido pelo Comodoro do Iate Clube da Paraíba, durante os meses de Março, Abril e Maio de 2010. Inicialmente, foi apresentado e assinado o Termo de Consentimento Livre e Esclarecido, seguido da aplicação do questionário e da entrevista.

As entrevistas foram gravadas, transcritas e, em seguida, submetidas ao soft Analysis of Qualitative Data (AQUAD) 6, o qual auxiliou na obtenção da frequência absoluta das palavras para construir as categorias analíticas. Estas foram apreciadas a partir da Técnica de Análise de Conteúdo, por ser um instrumento metodológico que dá suporte às análises das entrevistas, por meio dos procedimentos sistêmicos da descrição dos conteúdos (BARDIN, 2002). Para a construção dos diagramas que representam as categorias de análise foi utilizado o soft GoDiagram Express versão 2.6.2.

Ressalta-se que foram respeitadas as diretrizes regulamentadas pela Resolução ${ }^{\circ}$ 196/96 do Conselho Nacional de Saúde/MS, sendo o projeto aprovado pelo Comitê de Ética em Pesquisa com Seres Humanos da Universidade de Pernambuco, com o registro de número 231/09 e CAAE 0207.0.097.000-09.

\section{Resultados e Discussão}

As práticas corporais realizadas na natureza conduzem as emoções. Isso realça o gosto pela vida (que por vezes é enfraquecido e até perdido), permitindo ao praticante reencontrar uma intensificação nas sensações de compleição no mundo. No que se refere à prática da vela, as sensações podem potencializar o gosto de viver, o que proporciona o aumento da resiliência. Neste contexto, os resultados e a discussão estão apresentados em três categorias analíticas: sensações 
sem a prática da vela; sensações em situações de risco na prática da vela; e a resiliência na prática da vela.

\section{Sensações sem a prática da vela}

Esta categoria apresenta as sensações reveladas pelos velejadores entrevistados, destacando as sensações negativas quando não praticam a vela. Em relação a este aspecto, Valle (2007) aponta que o desenvolvimento da sociedade trouxe a vivência de situações estressantes, o que gera efeitos em vários níveis, e dificulta o exercício das atividades do cotidiano. O diagrama a seguir, ilustra as sensações sobre esta dimensão assinaladas pelos entrevistados.

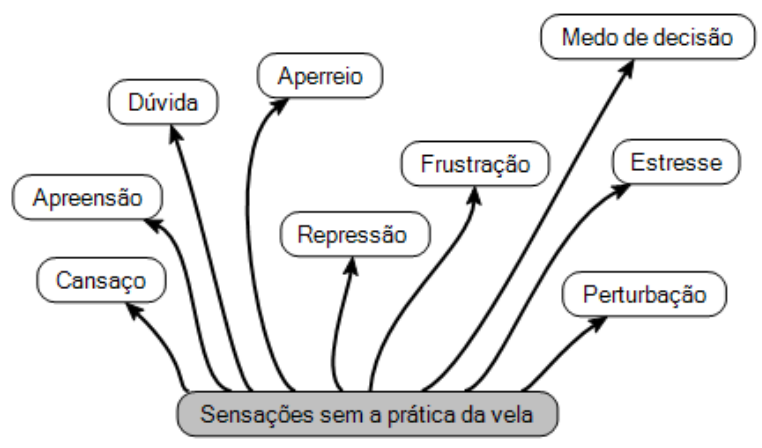

Diagrama 1: Características das sensações dos atores sociais sem a prática da vela.

A categoria Sensações sem a prática da vela, destacada na cor cinza, se refere às percepções provocadas pela ausência da prática da vela, e na cor branca estão as variáveis de maior frequência. As sensações negativas apontadas pelos entrevistados precisam ser controladas no cotidiano a partir de um modelo de processo de resiliência, focado na aquisição e no fortalecimento das qualidades resilientes, em um processo cíclico na perspectiva de mudança de vida e/ou adversidade (GALLI; VEALEY, 2007). Cabe realçar que as sensações negativas correspondem aos aspectos sociais e psicológicos, assim, os seres humanos precisam se adaptar a determinada circunstância (VALLE, 2007).

Os relatos dos atores sociais apontam que a prática da vela é um instrumento que faz parte do cotidiano, e sua ausência traz fatores psicossociais negativos. Como descreveu o entrevistado 9 , que quando não veleja tem a sensação de que "[...]falta alguma coisa na minha vida".

Nesta direção, Galli e Vealey (2007) ressaltam que o esporte proporciona a capacidade de desenvolver qualidades resilientes, o que oportuniza mudanças para o desenvolvimento pessoal. Assim, os entrevistados percebem a importância da prática da vela para o equilíbrio emocional e melhor desempenho nas atividades da vida cotidiana. 
Desta forma, as sensações negativas relacionadas ao cansaço, perturbação, aperreio e estresse foram apontadas pelos participantes, como relatou o entrevistado 17: "Quando eu saio da água, eu volto totalmente desestressado [...] e os problemas vão surgindo no dia a dia e o estresse vai voltando". Vale ressaltar que o entrevistado se refere à prática da vela no âmbito recreativo, sendo um fator positivo para a diminuição do estresse. Estudos prévios apontam que o estresse é um dos elementos chave para compreender o risco e a resiliência, incluindo fatores de proteção como redutores de experiências estressantes (SAPIENZA; PEDROMÔNICO, 2005; HAGGERTY, et al, 2000). Outras investigações indicam que a prática do lazer promove equilíbrio na vida, o que facilita a resiliência e a capacidade de combater o estresse (IWASAKI, et al., 2010; ALLEN; HURTES; STEVENS, 1999).

No que se refere às variáveis pautadas no medo de decisão, apreensão e dúvida, os participantes revelaram que a prática da vela ajudou a melhorar o seu poder de decisão, justificado pelo fato de que existem situações que precisam de tomada de decisão imediata, a fim de evitar acidentes. Neste aspecto, pesquisas assinalam que a prática esportiva pode despertar sensações positivas no que refere ao poder de decisão, como a autodeterminação e a motivação (DECI; RYAN, 2000; TAYLOR et al, 2010; SABISTON, et al., 2010).

As variáveis catalogadas como frustração e repressão, também foram apontadas pelo entrevistado 14: "[...] quando eu não velejo, eu me sinto frustrado". Estas sensações podem ser minimizadas a partir do engajamento em programas de recreação, promotores de mudanças nas atitudes resilientes. Isso foi verificado por Hurtes et al. (2000), onde a recreação se apresenta como uma intervenção do desenvolvimento em si, capaz de incorporar manifestações resilientes, adequadas e eficazes no tocante a fatores como: responsabilidade social, satisfação, gerência da raiva, entre outras habilidades específicas. Nesta direção, Allen, Hurtes e Stevens (1999) também investigaram o significado social e os fatores da melhoria dos níveis da resiliência, por meio de práticas de lazer e recreação, apontando para benefícios psicossociais dos sujeitos.

Diante deste cenário, a ausência da prática da vela no âmbito do lazer desperta sensações negativas, que interferem no relacionamento familiar e no trabalho dos sujeitos, provocando desequilíbrio emocional. Assim, os mesmos podem estar vulneráveis à predominância de sensações negativas.

\section{Sensações em situação de risco na prática da vela}

A categoria referente à situação de risco, assinalada na cor cinza, advém das sensações que os velejadores enfrentaram em circunstâncias vulneráveis, por meio das incertezas da natureza, focando o vento e o mar. Seguidas da categoria, estão as variáveis de maior relevância para os atores sociais, marcadas na cor branca, que recaem nas sensações negativas, como ilustra o diagrama a seguir. 


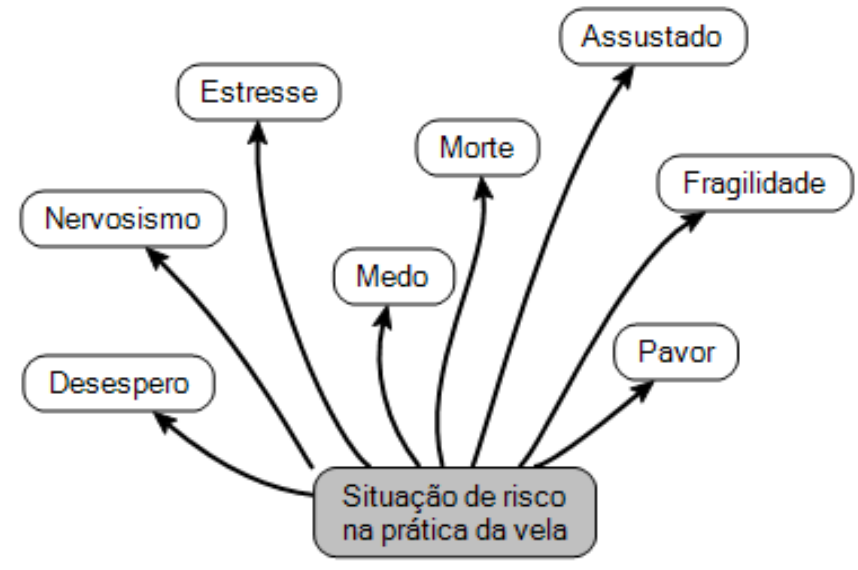

Diagrama 2: Sensações em situação de risco na prática da vela.

A paixão pelo risco, objetivando o prazer nas práticas corporais realizadas na natureza, envolve o esforço físico além dos próprios limites, passando por exaustão, fome, sede, frio, dúvida e medo, onde o sujeito se encontra em um fio condutor, em que o esforço e a eventualidade de um acidente se fazem presentes (LE BRETON, 2009; 2007). Contudo, o autor aponta que o número de vítimas nas práticas realizadas na montanha e no mar é crescente. Nesta direção, os dados coletados vão de encontro a esta afirmação, no tocante à prática da vela, sendo que apenas um entrevistado não vivenciou situação de risco.

As principais ocorrências de acidentes dizem respeito à falta de manutenção e quebra de equipamento, mudança climática e falta de experiência, como mostra o entrevistado 3: “[...]caí da prancha, quando eu fui submergir, a vela estava sobre minha cabeça. Eu fiquei apavorado e por fração de segundos eu mergulhei de novo e saí mais adiante, saí do sufoco [...] a gente pensa até na morte". Neste sentido, Le Breton (2009) aponta as situações de risco nas práticas corporais realizadas na natureza que conduzem ao jogo de morte, e a intenção não é de morrer, mas de intensificar o gosto pela vida e o ressignificado de si. Outros dados constam nos estudos de Rabinowitz e Frauman (2009), que examinaram as tendências pela busca de sensações em atividades de risco na natureza, e encontraram que a disposição dos jovens surge a partir da busca de fortes emoções e do espírito de aventura.

Nesta perspectiva, a busca por fortes emoções está ligada à vulnerabilidade do risco, no sentido de que as piores sensações nos momentos de perigo podem ser superadas, e as consequências negativas, como problemas psicossociais, podem ser evitadas, melhorando os fatores de resiliência. Para melhor compreender as situações de risco, Le Breton (2006) afirma que tudo o que passa pelo corpo é

Pensar a Prática, Goiânia, v. 17, n. 1, p. 01-294, jan./mar. 2014 
valorizado, como a resistência, a luta, a força, o esforço e o suor, levando o sujeito ao limite. Assim, essa valorização do sujeito possibilita o aumento da autoestima, a superação, a positividade com relação a si mesmo, a responsabilidade, a criatividade e outros fatores psicológicos.

Seguindo este raciocínio, o entrevistado 2 descreveu momentos de fortes emoções e como estas sensações se aproximam do risco no windsurf, ao mostrar que:

Você procura o limite máximo, e realiza a manobra mais radical, que você pula mais alto e dá um giro no ar, ficando de cabeça pra baixo. Então, quando o vento está muito forte e você quer tentar uma manobra nova, você está sujeito ao erro. E são nessas situações que a gente erra e leva as pancadas no tronco, na cabeça... mas isso faz parte do esporte.

Estas vivências relatadas pelo entrevistado são importantes para o equilíbrio emocional, o que promove a redução das ocorrências de erros, bem como o conhecimento para sair da situação de risco. Contudo, os estudos de Silva e Freitas (2010) apresentam os riscos reais, que na maioria das vezes são provocados por negligência, podendo ocasionar lesões, acidentes, doenças e até casos fatais.

Diante das considerações, as sensações negativas em situação de risco na prática da vela acarretam condutas simbólicas de morte. Contudo, as sensações de risco alimentam a confiança em si, valorizando as próprias habilidades e capacidade de superar momentos de tensão e pavor.

\section{A resiliência na prática da vela}

As práticas corporais realizadas na natureza são importantes para o domínio de habilidades, principalmente em situações de risco (seja real ou imaginário), promovendo mudanças de comportamento, além de desenvolverem fatores de resiliência. A categoria Resiliência na prática da vela, destacada na cor cinza, apresenta as variáveis sobre os fatores resilientes, assinalados na cor branca, lembrados pelos velejadores entrevistados, como ilustra o diagrama a seguir. 


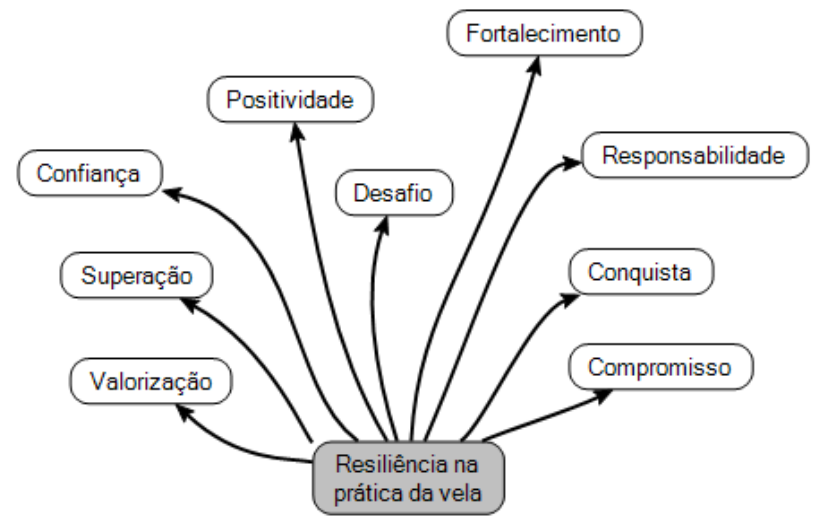

Diagrama 3: Fatores de resiliência por meio da prática da vela.

Os estudos de Iwasaki, Mactavish e Mackay (2005) apontam que as práticas de lazer melhoram os aspectos físicos, psicológicos e emocionais, o que facilita a sensação de equilíbrio emocional e da vida, bem como os níveis de resiliência. Desta forma, as situações de risco na prática da vela, no âmbito do lazer, auxiliam nas revelações e desenvolvimento pessoal, bem como no equilíbrio emocional, tanto nas situações de risco na prática da vela, como nas ocasiões da vida cotidiana.

Nesta direção, o entrevistado 11 relata uma situação de risco, em que o equilíbrio emocional favoreceu um melhor desempenho:

[...] estávamos voltando de Noronha quando a vela mestre se rasgou, prejudicando todo o equilíbrio do barco. Foi à noite, estava chovendo, e relampagueando, e eu e um colega tivemos que ir emendar a vela. Foi sufoco pra baixar aquela vela, amarrar toda, costurar, e tudo ali, enquanto os outros tentavam manter o barco equilibrado. [...] Em momento nenhum eu pensei: ah o barco vai virar, ah nós vamos naufragar. [...] O sentimento tem que vir na frente, e o que tinha era a minha capacidade de tirar aquela vela, remendar e levantá-la novamente.

Diante destas considerações, os desafios ao ar livre recaem nas experiências associadas ao equilíbrio emocional. Moore, Gerald e Wodarski (1997) ressaltam que a aventura conduz a aprendizagens e experiências que facilitam o desenvolvimento de competências. Além disso, os autores apontam que essas competências desenvolvidas a partir das experiências pelas práticas corporais realizadas na natureza são inerentes às atividades cotidianas, destacando as habilidades de comunicação, cooperação, tomada de decisão e resolução de problemas. 
Os fatores de proteção e resiliência nas práticas corporais realizadas na natureza facilitam desenvolver um sentimento de pertencimento e integração em situações de risco (BLOEMHOFF, 2006). Nesta perspectiva, o reconhecimento que o sujeito tem da própria valorização na realização de atividades em situações de risco, torna importantes os fatores de autoconhecimento e autoestima, possibilitando a conquista de autonomia em momentos de desafios, que muitas vezes são intransponíveis. Assim, a tripulação na prática da vela, em situações de risco, precisa estar equilibrada emocionalmente e ciente das circunstâncias, atestando a resistência ativa no sentido de converter o quadro de perigo.

As variáveis apresentadas no diagrama 3 apontam para as transformações das experiências pela prática da vela, lembradas pelos velejadores entrevistados. Bloemhoff (2006) ressalta que, quando solucionadas as situações de risco, os valores são reforçados, aumentando os fatores de proteção e competência. Desta forma, o entrevistado 19 mostra que a prática da vela trouxe "uma mudança enorme de responsabilidade, de disciplina e de ajudar o próximo". Nesta direção, as experiências pela prática da vela reforçam a condução arriscada dos comportamentos, sendo melhorados a partir das sensações positivas e dos fatores de resiliência.

Sob esta dimensão, a prática da vela desvenda valores pessoais que contribuem para o aumento da resiliência. Além disso, os desafios enfrentados no mar contribuem para o trabalho em grupo, favorecendo o respeito das escolhas de ideias, a integração e relacionamento da tripulação. Assim, as condutas na prática da vela envolvem a confiança grupal, o respeito, a responsabilidade e as iniciativas de resolução de problemas, que necessitam da cooperação e participação de todos os membros da tripulação a bordo.

\section{Conclusão}

O estudo apresenta uma abordagem qualitativa à luz da temática da prática da vela e a resiliência. Propõem-se, a partir das experiências de situações de risco, transformações nos fatores resilientes, no tocante à capacidade dos velejadores de saber lidar com as situações adversas em momentos de dificuldade. Desta forma, a superação das circunstâncias aponta para os fatores de resiliência.

Nesse sentido, a busca do prazer por meio do risco na prática da vela desperta para a capacidade de desenvolver fatores de proteção que influenciam na minimização dos fatores negativos e dos estressores ambientais. Vale destacar que a resiliência representa um outro olhar para as situações adversas, trazendo um ressignificado para o momento, e não a eliminação das sensações negativas, que estão inseridas no contexto histórico-social.

As situações de risco na prática da vela foram observadas como um dos fatores que podem auxiliar na melhoria da resiliência. No entanto, são necessários 
elementos relacionados aos aspectos psicológico, social, cultural, econômico, segurança, entre outros, para promover a superação em ocasiões adversas.

Diante da escassez de estudos que tratam da resiliência e da prática da vela, torna-se necessário o desenvolvimento de novas pesquisas que aprofundem e tragam resultados com atores sociais oriundos de outras regiões, faixas etárias e gênero, que vivenciem a situação de risco.

Em suma, os velejadores encontraram na prática da vela uma atividade onde, mesmo em momentos de risco e situações adversas, eles são capazes de solucionar problemas, obtendo melhorias nos fatores de resiliência, e valorizando suas habilidades e competências. Além disso, tais situações conduzem a uma redução das sensações de estresse frente aos problemas encontrados no cotidiano.

\title{
RISK AND RESILIENCE PSYCHOSOCIAL: CHALLENGES IN PRACTICE OF SAIL
}

\begin{abstract}
The aim of this study was to analyze factors of resilience from a hazard in the practice of sail. The field research involved 19 sailors of Iate Clube da Paraíba, aged 19 to 64 years. Was used in data collection a sociodemographic questionnaire and a schedule of semistructured interviews, which were transcribed and subjected to soft AQUAD 6, to obtain categories based on content analysis, being organized in diagrams. The results pointed to three categories: feelings without practice of the sail; feeling at risk in the practice of sail; and resilience in the practice of sail. It was observed that in this practice, there has been progress in emotional, bringing new meanings to life. Thus, the emotional balancehelps in situations of risk found the sea led by improving the factores if resilience.
\end{abstract}

Keywords: Risk. Resilience. Sail.

\section{RIESGOS PSICOSOCIALES Y RESILIENCIA: DESAFÍOS EN LA PRÁCTICA VELA}

\section{Resumen}

El objetivo del estudio fue analizar los factores de la resiliencia de las situaciones de riesgo en la práctica a vela. La investigación de campo participan 19 regatistas del Iate Clube de Paraíba, con edades entre 19 a 64 años. Fue utilizado en la recolección de datos un cuestionario sociodemográfico y de entrevistas semi-estructuradas, que fueron transcritas y sometidas a soft AQUAD 6, para obtener la categoria basado en el análisis de contenido, organizado en diagramas. Los resultados apuntaron a tres categorías: sin yachting sensaciones, sensaciones en situación de riesgo en la vela, y la resistencia en la vela. Se observó que esta práctica no había avançonos factores emocionales, trayendo un ressiginificado para la vida. Por lo tanto, el equilibrio emocional ayuda en situaciones de riesgo en contrados en el mar llevada a cabo por la mejora de los factores de resiliencia.

Palabras clave: Riesgo. Resiliencia.Vela. 


\section{Referências}

ALLEN, L.; HURTES, K. STEVENS, B.Making an impact. Parks \& Recreation, v. 34, n. 11, p. $68-77,1999$.

BANKS, M. Dados visuais para pesquisa qualitativa. Tradução J. Fonseca. Porto Alegre: Artmed, 2009.

BARDIN, L. Análise de conteúdo. Tradução Luís Antero Reto e Augusto Pinheiro. Lisboa: Edições 70, 2002.

BAUDRILlARD, J. A Sociedade de Consumo.Lisboa: Edições 70, 2008.

BLOEMHOFF, H. J. The effect na adventure-based recreation programme (ropes course) on the development of resiliency in AT- risk adolescent boys confined to a rehabilitation Center. South African Journal for Research in Sport, Physical Education\& Recreation (SAJR SPER), v. 28, n. 1, p. 2-12, 2006.

BRANDT, R.; VIANA, M. S.; SEGATO, L.; ANDRADE, A. Estados de humor de velejadores durante o Pré-Panamericano. Motriz, v. 16, n. 4, p. 834-840, out./dez. 2010 .

BRUHNS, H. A busca pela natureza: turismo e aventura. Barueri: Manole, 2009.

COHEN, L. MANION, L.;MORRISON, K. R. B.Research methods in education. $5^{\circ} \mathrm{Ed}$. Londres: Routledge, 2000.

COOKE, F. J.; SABIN, C.; ZUCKERMAN, J. N. A study of the incidence of accidents occurring during an Artic Expedition: another important aspect of travel medicine? J. Travel Med, v. 7, n. 4, p. 205-207, 2000.

DECI, E.L.;RYAN, R.M. The "what" and "why" of goalpursuits: Human needs andtheself-determination of behavior. PsychologicalInquiry,v. 11, p. 227-268, 2000 .

DELGADO, J.; GUTIÉRREZ, J. Métodos y Técnicas Cualitativas de Investigación em Ciencias Sociales.Madrid: Editorial Sínteses, 1995.

DOLBIER, C. L.; JAGGARS, S. S.; STEINHARDT, M. A. Stress-related growth: pre-intervention correlates and change following a resilience 
intervention.Stress \& Health: Journal of the International Society for the Investigation of Stress,v. 26, n.12, p135, 2010.

FERNANDES, H. M.; BOMBAS, C.; LÁZARO, J.; VASCONCELOS-RAPOSO, J.; Perfilpsicológico e sua importânciano rendimento em vela. Motricidade, v. 3, n. 3, p. 24-32, jul. 2007..

FRASER, E.; PAKENHAM, K. I. Resilience in children of parents with mental illness: Relations betweenmental health literacy, social connectedness and coping, and bothadjustment and caregiving. Psychology, Health \& Medicine, v. 14, n. 5, p. 573-584, 2009.

GALLI, N.;VEALEY, R. S. "Bouncing back" from adversity: Athletes'experiences of resilience. The Sport Psychologist, v. 22, p. 316-335, 2008.

GALLI, N.;VEALEY, R. S. Thriving in the face of adversity: Applying the resiliency model to high-levelathletes. Journal of Sport \& Exercise Psychology, v. 29, jul supplement, 2007.

GOULD, D.; DIEFFENBACH, K.; MOFFATT, A. Psychological characteristics and their development in Olympic champions. Journal of Applied Sport Psychology, v. 14, n. 3, p. 172-204, 2002

GREEN, G. T.; KLEIBER, D. A.; TARRANT, M. A. The effect of an adventurebased recreation program on development of resiliency in low income minority youth. Journal of Park \& Recreation Administration,v. 18,n. 3. p. 76-97, 2000.

HAGGERTY, R. J., SHERROD, L. R., GAMEZY, N. \&RUTTER, M. Stress, risk and resilience in children and adolescents: process, mechanisms and interventions. New York: CambridgeUniversity Press, 2000.

HENDERSON, K. Dimensions of choice: a qualitative approach to recreation parks and leisure research. State College, PA: Venture Publishing, 1991.

HOLLINSHEAD, K. The shift to constructivism in social inquiry: some pointers for tourism studies. Tourism Recreation Research,v. 31, n.2, p. 43-58, 2006.

HURTES, K. P.; ALLEN, L. R.; STEVENS, B. W.; LEE, C. Benefits-based programming: making an impact on youth. Journal of Park \& Recreation Administration Spring, v. 18, n. 1, p. 34-49, 2000. 
IWASAKI, Y.; MACTAVISH, J.; MACKAY, K. Building on strengths and resilience: leisure as a stress survival strategy. British Journal of Guidance \& Counselling, v. 33, n. 1, fev., p. 81-101, 2005.

LACHARITE, C. From risk to psychosocial resilience: conceptual models and avenues for family intervention. Texto contexto - enferm.,v.14, p. 71-77, 2005.

LAVOURA, T. N.; ZANETTI, M. C.; MACHADO, A. A. Os estados emocionais e a importância do treinamento psicológico no esporte. Motriz, v. 14, n. 2, p. 115123, abr./jun., 2008.

LAVOURA, T. N.; SCHWARTZ, G. M.; MACHADO, A. A. A democratização das atividades de aventura na natureza: o projeto "Canoagem Popular". Motriz, v. 13, n. 2, Rio Claro, p. 80-85, 2007.

LE BRETON, D. Condutas de risco: dos jogos de morte ao jogo de viver. Campinas: Autores Associados, 2009.

LE BRETON, D. A Sociologia do Corpo. $2^{\circ}$ ed., Petrópolis: Vozes, 2007.

LINCOLN, Y;GUBA, E. Paradigmatic controversies, contradictions and emerging confluences. In Denzin, N.; Lincoln, Y. (Eds.), The Landscape of Qualitative Research: Theories and Issues (2nd ed.). Thousand Oaks: Sage, 2003.

MARINHO, A. Lazer, Aventura e Risco: reflexões sobre atividades realizadas na natureza. Movimento,v. 14, n. 02, Porto Alegre, p. 181-206, 2008.

MARINHO, A. Lazer, aventura e ficção: possibilidades para refletir sobre atividades realizadas na natureza. Motriz, v. 15, n. 1, Rio Claro, p. 01-12, 2009.

MOOTE, G.T.; WODARSKI, J.S. The acquisition of life skills through adventurebased activities and programs: A review of the literature. Adolescence, v. 32, n. 125,p. 143-167, 2007.

PEACOCK-VILLADA, P.; DECELLES, F.; BANDA, P. S. Grassroot soccer resiliency pilot program: building resiliency through sport-based education in Zambia and South Afriaca. New Directions for Youth Development, v. 1, n. 116, 2007..

RABINOWITZ, E.; FRAUMAN, E. D. A preliminary investigation of sensationseeking and risk-based recreation among college students: implications for intramural and outdoor programs. Recreational Sports Journal, n. 33, p. 109-118, 2009. 
SABISTON, C.M.; BRUNET, J.; KOWALSKI, K. C.; WILSON, P. M.; MACK, D. E.; CROCKER, P. The role of body-related self-conscious emotions in motivationg women's physical activity. Journalof Sport \& Exercise Psychology,v. 32, p. 417-437, 2010.

SAPIENZA, G.; PEDROMÔNICO, M. R. M. Risco, proteção e resiliência no desenvolvimento da criança e do adolescente. Psicologia em Estudo, v. 10, n. 2, p. 209-216, mai./ago., 2005.

SILVA, P. P. C.; FREITAS, C. M. S. M. Emoções e riscos nas práticas corporais na natureza: uma revisão sistemática. Motriz. Rio Claro, v. 16, n. 1, p. 221-230, 2010 .

TAYLOR, I. M.; NTOUMANIS, N.; STANDAGE, M.; SPRAY, C. M. Motivational predictors physical education student's effort, exercise intentions, and leisure-time physical activity: a multilevel linear growth analysis. Journalof Sport \& Exercise Psychology, 32, p. 99-120, 2010.

VALLE, M. P. Coaching e resiliência: intervenções possíveis para pressões e medos de ginástica y esgrimistas. Revista Brasileira de Psicologia do Esporte, v. 1, n. 1, São Paulo, dez. 2007.

WALLER, M.A. Resilience in ecosystem context: Evolution of the concept. American Journal of Orthopsychiatry, v. 71, n. 3, 290-297, 2001.

Recebido em: 22/09/2012

Revisado em: $17 / 12 / 2012$

Aprovado em: 13/02/2013

Endereço para correspondência:

laprisci@gmail.com

Priscilla Pinto Costa da Silva

Universidade Federal da Paraíba

Cidade Universitária

João Pessoa - PB - Brasil

CEP: $58051-900$ 